\title{
Human cognitive and perceptual motor response: an integration of idiothetic and allothetic processes
}

\author{
G. Silverman \\ Department of Electrical and Computer Engineering, \\ Manhattan College, Riverdale, New York, U.S.A.
}

\begin{abstract}
The ability to quantify human behaviours is a foundation for being able to develop computer programs emulating intelligence (i.e., Artificial Intelligence (AI)). The implications for understanding intelligence can aid in the development of assist devices for medical intervention (e.g., Rehabilitation Medicine) and as models for robotic devices. Actions taken by human subjects are an integration of internal stimuli (idiothetic) and external (allothetic) stimuli. Learned (new) behaviours originate from existing behaviours. Complex skilled human behaviour is described as an integration of simple skills. An experiment is described to study this phenomenon. As modelled here, cognitive motor behaviour requires a subject to view a series of stimuli and make appropriate responses. Overall behaviour is modelled as a combination of a Memory process and a Response (Motor) process. Response latency, learning records are derived from this motor learning experiment. A non-linear model is proposed and a Monte-Carlo simulation is used to evaluate consistency with the experimental results.
\end{abstract}

Keywords: cognitive science, deliberative learning, motor response, stochastic model of cognitive motor behaviour.

\section{Introduction}

Cognitive science is the interdisciplinary study of the mind. Its primary methodology is scientific although many additional methods contribute, including those from philosophy, psychology, linguistics, AI, robotics and neuroscience. Each of these endeavours carries with it a unique set of tools and 
perspectives. In order to appreciate a subject as complex as the mind, we need to approach its study from a unique theoretical perspective. This perspective centres on the idea of computation, and may alternatively be referred to as information processing; cognitive scientists view the mind as an information processor that manifests itself in behavioural responses particularly in human activities [1]. The ability to quantify human behaviours is a foundation for being able to develop computer programs emulating intelligence (i.e., (AI)). The implications for understanding intelligence can aid in the development of assist devices for medical intervention (e.g., Rehabilitation Medicine) and as models for robotic devices $[2,3]$.

\subsection{Human behaviour}

From one viewpoint, observable biological behaviour is a fundamental building block of natural intelligence. Behaviour is considered to be a mapping (translation) of sensory inputs into a pattern of motor actions whose purpose is to complete a given task. Behaviour has three well-defined categories. This is depicted in fig. 1 [1]. In some instances, responses and categories depend on the organism's memory, particularly previously stored patterns of behaviour.
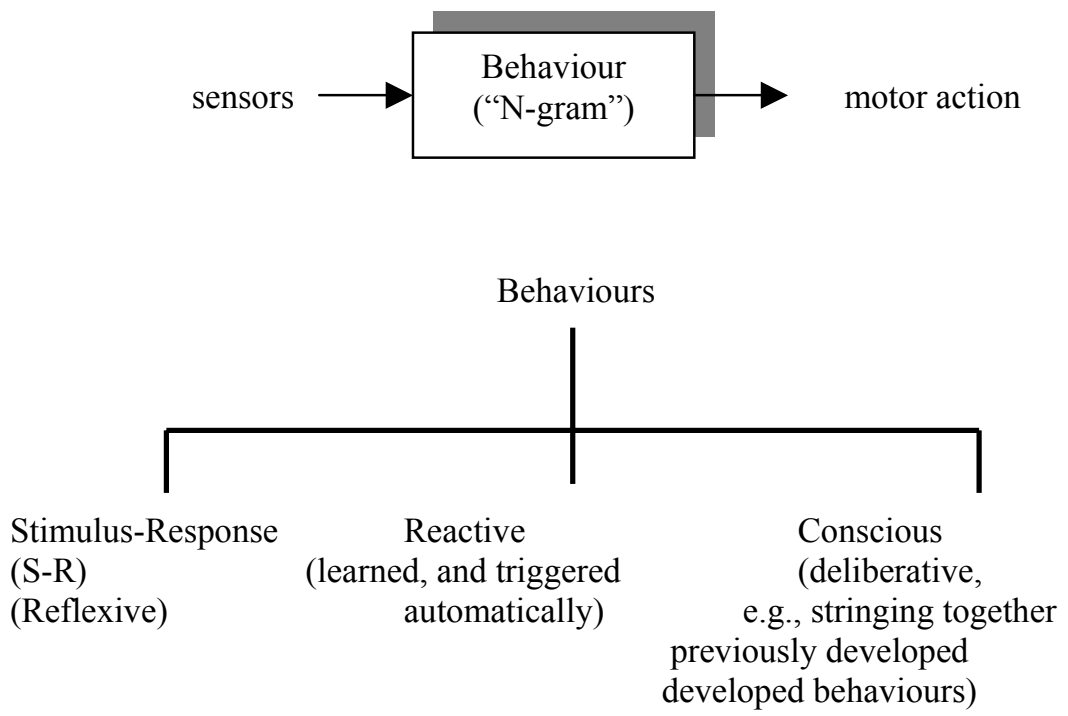

Figure 1: Classification of animal behaviours.

The "N-gram" is a map or transformation of the sensor data into motor action. $\mathrm{N}$-grams are representations of sequences of "elementary" actions that are stored in our brain such as the transformation of notes on a musical score into positions and actions of our fingers on a musical instrument. Reflexive responses last as long as the stimulus that produced them and the magnitude of the response is 
proportional to the stimulus intensity (e.g., removing one's hand from a hot surface). Reflexive behaviours further include fixed-action patterns of response that continues for a longer duration than the stimulus - consider cases where animals flee predators even though the danger may have ceased. Reactive responses are learned behaviours and consolidated such that they are executed without conscious thought. Subsets of such responses include taxes where the organism moves to a particular orientation. For example, hatching turtles move toward the ocean using reflected moonlight. Deliberative behaviour includes a conscious component where previously developed behaviours may be strung together. In summary, behaviour is a coupling of perception and action, an integration of external and internal stimuli. The ability to quantify animal behaviour suggests that computer programs can be written to reproduce such activities. The psychologists K. Lorenz, [4] and N. Tinbergen [5] pioneered ethology (the study of animal behaviour) and are considered to be the fathers of this scientific investigation. Through their work with terns and other animals, they identified four ways for an animal to acquire and organize components underlying behaviour:

- Innate: the animal is born with a single, comprehensive, behaviour (e.g., feeding behaviour)

- Born with a sequence of innate behaviours: each step in the behaviour sequence is triggered by the combination of the internal state of the animal and the environment in which it is functioning. (As an example, the mating cycle of digger wasps: mating, followed by nest building; followed by egg laying.)

- Innate with memory: represented as initial behaviours of the animal (at birth) but one that requires some initial stimulus to cement the behaviour. (Navigational behaviour of bees is an example of such behaviour. The baby leaves the hive for short distances; these distances are gradually increased.).

- Learned behaviours: a new behaviour evolves from existing behaviours (e.g., hunting is composed of sub-behaviours such as searching, stalking, chasing, etc.)

The models proposed by Lorenz and Tinbergen can be used to understand and simulate human behaviour, particularly as it relates to processing of information that an individual may not have previously encountered. In such cases, initial behaviours are deliberative in character and, as subjects acquire the underlying knowledge, subsequent stimuli produce reflexive responses. This model closely follows representations of learned behaviours that evolve from existing behaviours. When presented with a new or novel task, acquisition of the skill or required knowledge proceeds by serial chaining [6]. Initially, such learning is governed by input stimuli. As learning proceeds, a given stimulus is accompanied by feedback (idiothetic) stimuli produced by previous responses. A typical example of such learning is demonstrated by what happens when a human subject $(\mathrm{S})$ learns to memorize a poem. When $\mathrm{S}$ is asked to recite a particular line of the poem (e.g., line 7), the latency to the start of recitation is a 
linear function of the line number; each verbalized line is the stimulus for the next.

\section{Methods}

A sequence of stimuli is presented to Ss who make a motor response at a location that corresponds to the position of the visual stimulus. A personal computer (PC) was used to generate the stimuli and to record latency of each $\mathrm{S}$. To reveal underlying behaviours, one group of Ss received a pure "unstructured" random sequence while other groups were tested using patterned sequences (i.e. repeated groupings). No sequence was patterned in a way that made the sequence obvious after only a few stimuli presentations (e.g., 1, 3, 5, and 7 ...where the numbers represent response positions on the manipulanda - see fig. 2 below).

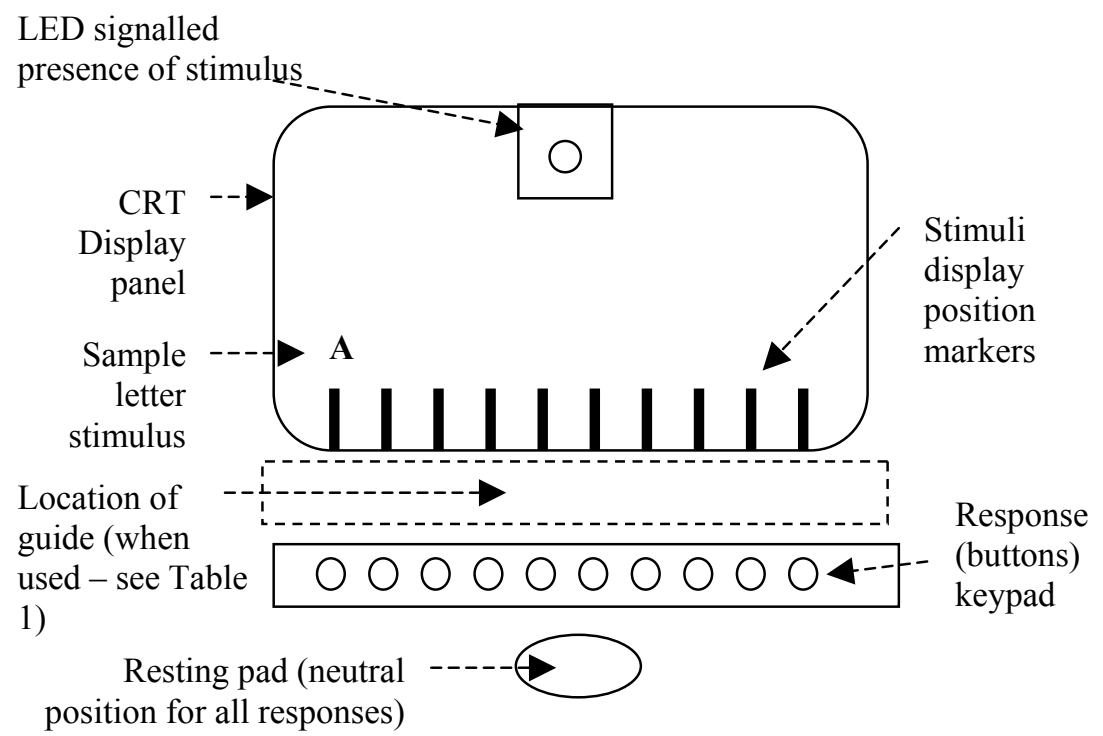

Figure 2: Sketch of interactive panel to study cognitive-motor learning behaviour

A series of six different types of tests were performed. These were designed with varying "information" content. (See Table 1 below.) Each test included a group of six Ss (for a total of $36 \mathrm{Ss}$ ). The Ss who performed this experiment did not comprise a homogeneous group. Some were college students, while others included Ss obtained through a state employment service. No attempt was made to differentiate responses based on varying backgrounds. Each test was composed of a repetition of 12 trials (epochs) and consisted of a sequence of either 9 or 18 alphanumeric characters displayed on the display panel. A trial was 
followed by a one-minute rest interval. After the fifth trial, the $\mathrm{S}$ was given a five-minute rest period. Each character that was displayed had an inter-trial delay period of 0.9 seconds, a stimulus interval of 0.9 seconds, and a display period of 0.99 seconds. (The total character presentation was 1.89 seconds.) This is considered sufficient to allow learning to take place (as opposed to the perception that this was a "tracking" experiment that would not exhibit learning). The tests for each $\mathrm{S}$ were arranged as follows (Table 1):

Table 1: $\quad$ Summary of experimental trials.

\begin{tabular}{|c|l|}
\hline $\begin{array}{c}\text { Trial } \\
\text { numbers }\end{array}$ & \multicolumn{1}{|c|}{ Notes } \\
\hline \multirow{1}{*}{1,2} & $\begin{array}{l}\text { Non-repeated sequence of characters - all 'X's - was displayed at } \\
\text { various locations on the display. This familiarized the S with the } \\
\text { apparatus; it also established a base line of response distributions, } \\
\text { as well as the average reaction times for unlearned events. The } \\
\text { PC generated the sequence of random positions on the display. }\end{array}$ \\
\hline Test group I: Ss received only 18 randomly located characters, \\
each one being an "X." The PC generated the random numbers. \\
Test group II: Nine-item Learning: The 9-item list was formed in \\
an arbitrary sequence, arranged to avoid predictive runs. The \\
sequence used was 6 20 3 5 7 1 8 4. (In a 9-item sequence all 10- \\
display positions was not possible.)
\end{tabular}




\subsection{Test results}

A good deal of data was extracted from the various tests. While a complete presentation of results is beyond the limitations of this paper, two outcomes are noted below because they - as with all six types of tests - confirm the simulation that was developed (see Section 3). Shown below are two characteristics results for the HM-CVC test: a comparative learning curve (fig. 3); a response latency graph (fig. 4).

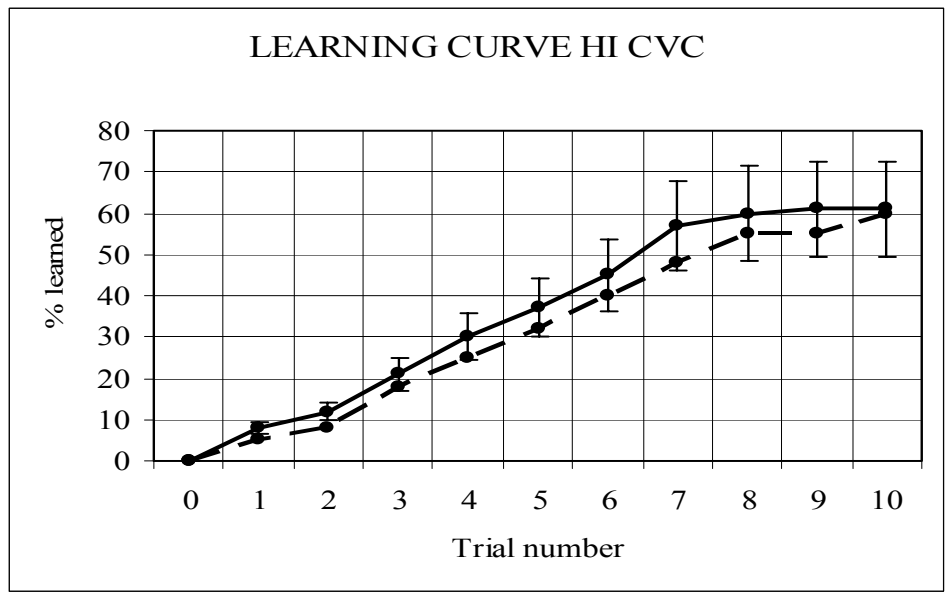

Figure 3: Learning results for HM-CVC experiment.

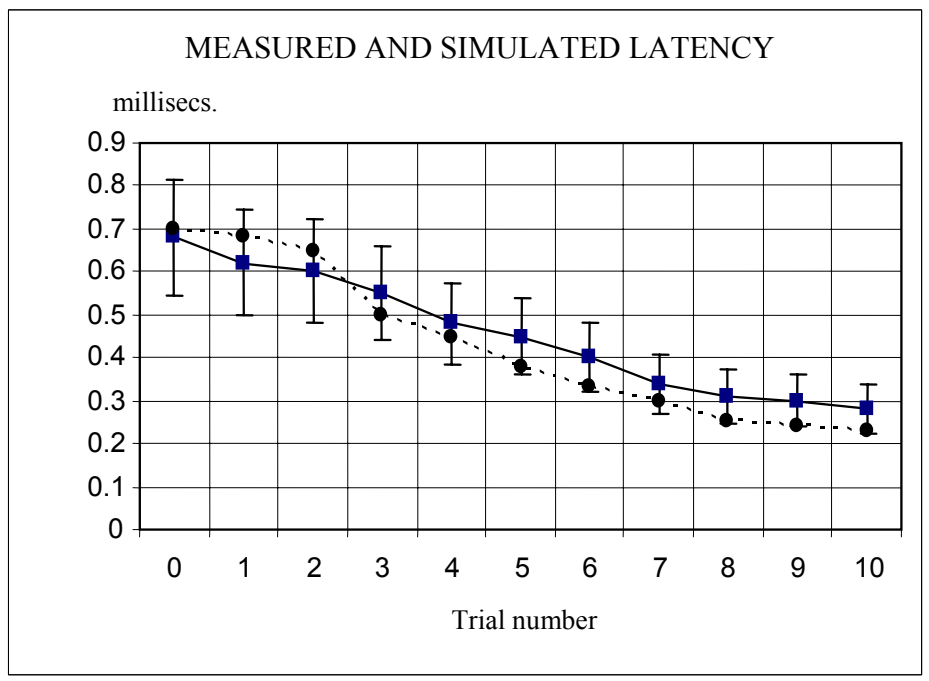

Figure 4: Latency results. 
(In both figs. 3 and 4, the solid curve represents measured results while the dashed curve comes from simulated data.)

\section{A stochastic model of cognitive motor behaviour}

A description of cognitive motor behaviour, particularly when a $\mathrm{S}$ is presented with a new learning task, is consistent with an appropriate combination of two behavioural processes ("subprograms"). The first is called the Response Process. It provides for a behavioural mechanism that simulates the time that it takes $\mathrm{S}$ to respond to a given stimulus. It contains a stochastic generator, so that the resultant reaction time distributions simulate observable behaviour patterns. A second (cognitive) process - called a Memory Process - represents the mechanism that underlies the way in which a $\mathrm{S}$ gradually acquires (learns) the organization of a train of repeated stimuli. The Flow Diagram for this cognitive motor simulation is shown in fig. 5 .

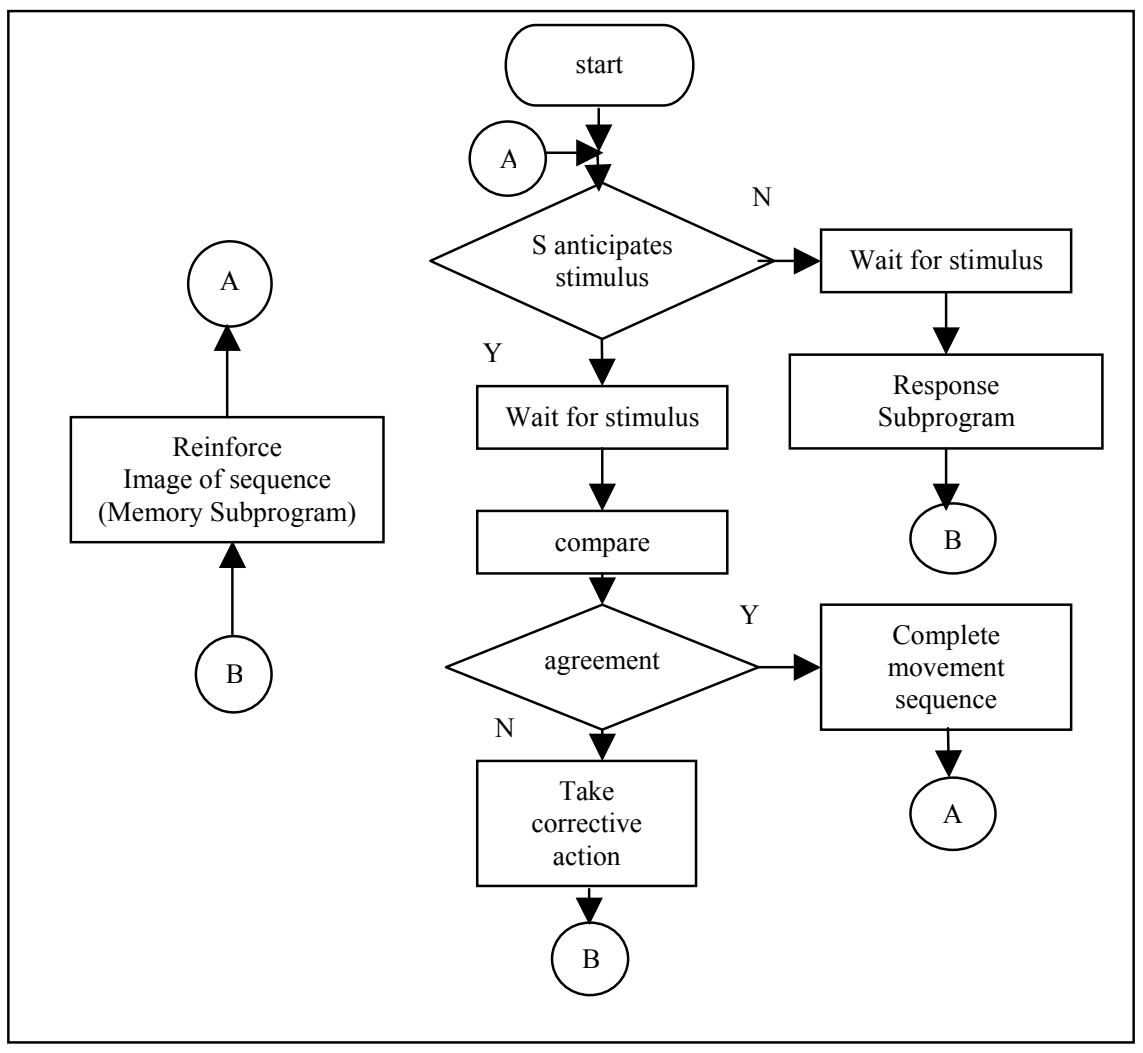

Figure 5: Flow diagram for cognitive motor simulation. 
This model is based on a time axis for each stimulus event that consists of three definitional periods. During an intertrial interval the simulated S completes cognitive tasks to determine if the next stimulus can be predicted. The stimulus is actually displayed during the stimulus interval. During a final "refractory" interval the stimulus has been turned off and the simulated $\mathrm{S}$ completes the response (if it was not completed during the stimulus interval). At the start, the memory is scanned to determine if an image of the next stimulus has already been "learned." If present, movement towards the corresponding response position is accomplished where completion of a Motor Response is relatively simple. In some instances, comparison of the image stimulus and the actual stimulus results in a mismatch. This may occur because "noise" or other interferences have been introduced during the retrieval of the image from memory; corrective action is now required. The simulation - as well as $\mathrm{S}$ in the experiment - acts to reinforce the image of the sequence in accordance with the Memory Subprogram (process). The simulation treats the current stimulus as if it is not yet learned. When there is no knowledge of the stimulus a different procedure is followed. The program performs what is referred to as "tracking behaviour." That is, when the stimulus occurs, the program invokes the Response Subroutine that results in an appropriate response. Both the Memory Subprogram (process) and the Response Subprogram (process) are significant with regard to the relevance of the paradigm.

\subsection{The response process}

The Monte-Carlo simulation is represented as a two-speed servomechanism. The program compares the number that represents the current response position - the position at which the S "hand" is located - against the position at which a response is needed. If the error is larger in absolute value than a parametric quantity noted as the Criterion, then the program follows a "high speed" response. When the difference falls below the Criterion, the program simulates a slower response. McGill, wherein a counting process could simulate response latency, has used this paradigm $[8,9]$.

\subsection{The memory process}

The process of learning a sequence of stimuli involves the memory functions within brain. Several features of human brain organization are incorporated into this simulation and certain modifications and stochastic processes - Monte Carlo methods - are introduced to assure that the simulation is consistent with cognitive motor observations. Human memory function has been much studied. [1]. Fig. 6 depicts the memory model used in the current study. The model provides for a nine-cell memory element of storage locations. This reflects models used by M.J.A Howe [10] and G.A. Miller [11]. In addition, the model includes a long-term memory register. This long-term memory eventually contains the representation of the stimulus train. The Initial Processor - see fig. 6 - includes a stochastic generator that randomly selects one of the (nine) temporary storage locations to receive the incoming data and simulates the flow 
of past information, characteristic of this short-term temporary memory function. (Data can overwrite previously stored data.) Finally, a Scanner accounts for the stochastic way in which information is transferred from temporary to permanent memory.

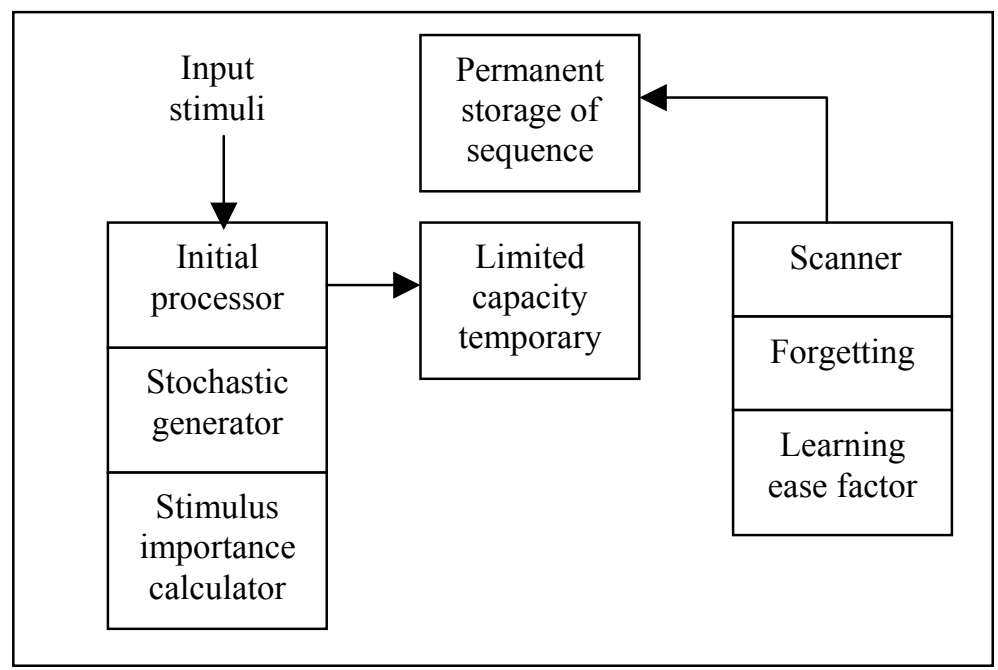

Figure 6: Memory model.

\section{Conclusions}

The stochastic processes that simulate a cognitive motor response task for a human $\mathrm{S}$ were able to predict both response latencies and stimulus acquisition. In part, distributions of response time latencies from the experimental population and the simulation were examined. A Chi Square analysis was used to test if both distributions came from the same population. The result produced a Chi Square value of 10.66, corresponding to 8 degrees of freedom. In accordance with standard practice, class values were grouped so that fewer than 5 occurrences did not appear in the calculation [12]. With 8 degrees of freedom, the rejection limit is 11.0. Since $10.66<11$, we do not reject the hypothesis that both distributions came from the same population.

When presented with a new or novel task, acquisition of the skill or required knowledge proceeds by serial chaining. Initially, such learning is governed by input stimuli. As learning proceeds, a given stimulus is accompanied by feedback (idiothetic) stimuli produced by previous responses. Results from a stochastic model of such cognitive motor situations are consistent with the underlying model. Such models have important implications for design of intelligent agents such as autonomous robots with the ability to emulate human cognitive capabilities. 


\section{References}

[1] Friedenberg, J., Silverman, G., Theories of Mind: An Introduction to Cognitive Science, Sage Publications, Thousand Oaks, CA, 2005.

[2] Silverman, G., Artificial intelligence in rehabilitation medicine: An emerging technology, Proc. International Conference on Artificial Intelligence and Applications, Marbella, Spain, Sept. 4-7, 2001

[3] Brooks, R. A., Flesh and Machines: How Robots Will Change Us, Pantheon Books, New York, N.Y., 2002.

[4] Lorenz, K. Man meets dog. Kondanska Globe, New York, New York, U.S.A., 1994.

[5] Tinbergen, N., (1972) Animal in its world, explorations of an ethologist 1932-1972, Harvard University Press, Cambridge, Massachusetts, U.S.A., 1972.

[6] Greenwald, A.G., Sensory feedback mechanism in performance control with special reference to the ideo-motor mechanism, Psychological Review, 77, 2, March 1970.

[7] Archer, E.J., Re-evaluation of the meaningfulness of all possible CVC trigrams, Psychological Monographs, 74, 497, 1960.

[8] McGill, W.J., Stochastic latency mechanism, Handbook of Mathematical Psychology (Luce, Bush, Galanter, (eds.)), vol. 1, John Wiley and Sons, Inc., New York, N.Y., 1963.

[9] McGill, W.J., Gibbon, J., The General-Gamma Distribution and Reaction Times, Journal of Mathematical Psychology, 2, 1-18, 1965.

[10] Howe, M.J.A., Introduction to human memory: A psychological approach, Harper and Row, New York, N.Y., 1970.

[11] Miller, G.A., The magical number seven, plus or minus two: some limits on our capacity for processing information, The Psychological Review, 63, 2, 81-97, March 1956.

[12] Lindgren, B.W., McElrath, G.W., Introduction to probability and statistics, The Macmillan Co., New York, N.Y., 1959. 